\title{
Desempeño cívico, compromiso organizacional y satisfacción vital en el voluntariado
}

Sandra González Checa, Shirley González, Pablo Linares Mingorance, Carlos Rentero Castro y Cristina Lucio Villegas de Cea. Universidad de Granada

\author{
Recepción: 31 de mayo de 2016 | Revisión: 15 de junio de 2016 | Aceptado: 12 de julio de 2016 \\ Correspondencia: sandritaglez@correo.ugr.es \\ Citar: González, S., González, S., Linares, P., Rentero, C. y Lucio, C. (2016). Desempeño cívico, compromiso organizacional y \\ satisfacción vital en el voluntariado. ReiDoCrea, 5(2), 63-70.
}

\begin{abstract}
Resumen: El propósito de esta investigación es encontrar en qué medida la participación en distintos tipos de voluntariado se asocia con mayores niveles de satisfacción vital, compromiso organizacional y desempeño cívico. La muestra del estudio consta de 49 participantes, voluntarios de protección civil, $36.7 \%$, un refugio de animales, $36.7 \%$, y un grupo de teatro, 26.5\%, con un rango de edad entre 19 y 58 años. Los resultados muestran que el tipo de voluntariado no mantiene relación con la puntuación en las tres variables, probablemente debido al tamaño de la muestra. Se encontró que a medida que aumenta la edad aumenta la puntuación en desempeño cívico y compromiso organizacional, y que existe una correlación positiva entre desempeño cívico y compromiso organizacional. No se encontraron diferencias con respecto al sexo en las distintas variables.
\end{abstract}

Palabras clave: Psicología Social | Voluntariado

Civic Performance, Organizational Commitment and Life Satisfaction in Volunteer Work

Abstract: The purpose of this research study is to determine the extent to which participation in different types of volunteer work is associated with higher levels of life satisfaction, organizational commitment and civic performance. The sample for the study consists of 49 participants, volunteers in civil defense, $36.7 \%$, an animal shelter, $36.7 \%$, and a theater group, $26.5 \%$, with an age range from 19 to 58 years old. The results show no correlation between type of volunteer work and the score on the three variables used, presumably due to the size of the sample. It was found that the older the person the higher the score on organizational commitment and citizenship performance. A correlation was also found between organizational commitment and civic performance. There were no differences between sexes in the variables.

Keywords: Social Psychology | Volunteering

\section{Introducción}

El voluntariado ha sido uno de los dominios más investigados y en los que se han obtenido avances más relevantes en el área de la Psicología del Trabajo y de las Organizaciones, siendo éste el núcleo central del presente estudio. Existen gran cantidad de investigaciones que construyen la definición de dicho constructo; un ejemplo es la de Penner (2002), que explica que el voluntariado es una conducta con intención de ayuda, planificada, no obligatoria, realizada en un tiempo determinado, y dentro del ámbito organizacional. Gaviria, Cuadrado y López (2009) añaden que la planificación del voluntariado implica la consecución de unas metas, la existencia de medios materiales y humanos, programación, división del trabajo, y supervisión y corrección de las tareas. Otros autores (Nelson, 1997) aseguran que en el voluntariado se obtienen recompensas, pero no de tipo material o monetario, como en un empleo, sino basadas en los intercambios interpersonales que se consiguen al realizarlo.

En la misma línea en la que se trata de hablar de la labor del voluntario y su valía dentro de la organización, debe ser destacada la diferencia entre dos conceptos diferentes: desempeño de tarea y desempeño cívico. El desempeño de tarea o task performance es entendido como el examen detallado de una conducta observable asociada con la ejecución de uno de los objetivos determinados por una unidad de trabajo, siendo habitualmente acuñado en estudios de ámbitos laborales. En cambio, esta investigación, se centra en el desempeño de un trabajo no reconocido 
formalmente, no remunerado, por lo que hablamos de otra dimensión diferente: el desempeño cívico o citizenship performance (Motowidlo y Schmit, 1999).

El desempeño cívico se define como la realización de un conjunto de conductas voluntarias que, generalmente, no son parte del rol formal laboral ni reconocidas explícitamente en los sistemas de compensación. En conjunto, estas conductas promueven el funcionamiento organizacional eficaz (Bateman y Organ, 1983; Organ, 1988; Organ y Ryan, 1995; Ployhart et al., 2006). En otras palabras, son conductas que contribuyen al mantenimiento del sistema social facilitando el logro de las metas organizacionales, los gestos que lubrican la maquinaria social, y que están dirigidos a crear un ambiente organizacional de apoyo social y psicológico en el que el núcleo técnico debe funcionar (Borman y Motowidlo, 1993; Witman, Van Rooy y Viswesvaran, 2010).

Junto con esta variable, se ha estudiado también el papel del compromiso organizacional. Este es definido por Porter, Streers, Mowday y Boulian en 1974 como la intensidad con la que el empleado se ve unido con su organización. Más adelante, en 1997, los autores Meyer y Allen configuran su Modelo de Compromiso Organizacional dividiendo el constructo en tres componentes: factores afectivos (apego del empleado con la organización), compromiso normativo (sentimientos de obligación, deber y responsabilidad moral) y compromiso de continuidad (cómo se percibe el costo asociado con el trabajo y tiempo invertidos). Dicho modelo inspiró la línea de investigación existente acerca de este constructo, aunque en este estudio se planteará como un constructo unifactorial.

En relación a lo anterior, se ha encontrado que el tiempo invertido en la organización es una variable que, junto con el compromiso organizacional, correlaciona positivamente con la satisfacción, la productividad y la sensación de utilidad del voluntario en su organización (Washko, 2006). Corroborando esto, otros autores señalaron que "el compromiso de los miembros a su organización es necesario para la supervivencia de ésta, ya sea una unión de comercio o una organización de voluntariado" (Catano, Kelloway y Pond, 2001).

Como era de esperar, se ha encontrado que el compromiso organizacional es mayor en los voluntarios que en los empleados, ya que los primeros tienen una mayor implicación a nivel personal con la organización. Ahora bien, el tipo de actividad o desempeño de tarea en la que estos voluntarios están involucrados da lugar a diferencias personales, situacionales y actitudes organizacionales. Por esta razón, en esta investigación, se ha tratado de confeccionar una muestra heterogénea en el tipo de voluntariado.

Otra de las variables principales con las que se han encontrado correlaciones con el voluntariado dentro de la literatura es la satisfacción vital, definida como el componente cognitivo del bienestar subjetivo; esto es, según Diener (1984), Veenhoven (1991) y Dávila de León y Díaz Morales (2005) la valoración que la persona hace de su vida en general o de aspectos específicos de esta en particular. Cabe destacar que este constructo es el que trata el modelo Ecológico-Social del Bienestar de Little (1999), que explica sus tres componentes: características del contexto, características estables de personalidad, y unidades mediadoras (metas). Las investigaciones de Mischel y Shoda, (1995) Diener et al. (1999) y Little (1999) concluyen que la satisfacción vital queda mejor explicada desde esta perspectiva integradora que por cada componente por separado (Díaz Morales y Sánchez-López, 2001). 
Otros estudios lograron demostrar que la satisfacción aumenta a medida que la persona deja de centrarse en sí misma (Salmela-Aro y Nurmi, 1997; Strack, Argyle, y Schwart, 1991). Este hecho permite afirmar que el voluntariado aumenta la satisfacción vital (Díaz Morales y Sánchez-López, 2001). Por ello, y tal y como realizaron Dávila de León y Díaz Morales en su investigación del voluntariado y satisfacción vital de 2005, el presente estudio seguirá la línea de las investigaciones que sí hallaron relación entre estos dos constructos. Estos mismos autores no encontraron diferencias con la edad al relacionarla con satisfacción y voluntariado, por lo que consideraron la posibilidad de que la primera esté más relacionada con la actividad del voluntario en sí misma que con los años de desempeño, otro de los factores que pretende estudiar la presente investigación. Sin embargo, cabe señalar que se ha encontrado que la relación entre los dos constructos es bidireccional y profundamente modulada por el tipo de voluntariado realizado. Por ello, se adoptará una clasificación que distingue entre tres tipos de voluntariado: protección civil, defensa animal, animación cultural.

\section{Objetivos}

Estudiar las posibles relaciones existentes entre los constructos desempeño cívico, satisfacción vital y compromiso organizacional en el ámbito del voluntariado, así como la relación de estos con varios tipos de voluntariado y otras variables personales y sociodemográficas.

\section{Hipótesis}

Existen diferencias entre el compromiso organizacional, la satisfacción vital y el desempeño cívico en las personas voluntarias, dependiendo del tipo de organización al que pertenecen. También se espera encontrar relación entre las tres variables señaladas, de modo que las personas participantes que puntúan alto en un constructo lo hagan también en otro. Por último, no se descarta encontrar relación entre las tres variables antes señaladas y las variables personales y sociodemográficas medidas.

\section{Método}

\section{Participantes}

El estudio ha sido realizado con una muestra de 49 miembros, pertenecientes a diversas organizaciones de voluntariado. La muestra está conformada por un $44{ }^{\prime} 9 \%$ de mujeres y un $551 \%$ de hombres, procedentes de distintas agrupaciones de Protección Civil de la provincia de Granada (Granada, Cijuela y Baza), 36'7\%, voluntarios destinados a la protección animal a través de las organizaciones Por Patas (Granada) y Ladridos Vagabundos (Pinos Puente), 36'7\%, y voluntarios dedicados a la animación cultural en el Grupo de Teatro y Poesía Prometeo (Granada), 26'5\%. La muestra se encuentra entre los 19 y los 58 años de edad $\left(M=28\right.$ '61; $\left.D T=10^{\prime} 91\right)$, todos ellos de nacionalidad española y residencia en Granada (España).

\section{Instrumentos}

A continuación se exponen los distintos instrumentos empleados para la medición de las variables.

En primer lugar, para medir la variable compromiso organizacional se ha utilizado el Cuestionario de Compromiso Organizacional de Meyer y Allen (1997). El cuestionario cuenta con una fiabilidad superior a 0.70. Esta versión consta de 12 ítems, con una 
escala tipo Likert de cinco niveles, siendo estas sus posibles respuestas: "1 (Muy en desacuerdo), 2 (En desacuerdo), 3 (Indeciso), 4 (De acuerdo) y 5 (Muy de acuerdo)". A modo de ejemplo, resaltar el ítem 8: <<Esta organización merece mi lealtad >>.

En segundo lugar, para medir la variable desempeño cívico se ha utilizado la adaptación al español de la Escala de Desempeño Cívico de Coleman y Borman (Díaz-Vilela et al., 2012). La fiabilidad del cuestionario es relativamente alta atendiendo al coeficiente de Cronbach $(\alpha=0.96)$. Esta versión consta de 27 ítems, siguiendo el mismo formato que el cuestionario anterior. La versión cuenta con elementos como $<<$ Soy entusiasta y persistente en mi trabajo >> correspondiente al ítem 1.

En tercer lugar, para medir la variable satisfacción vital se ha utilizado la adaptación al español de la Satisfaction With Life Scale (SWLS) (Diener et al., 1985). La fiabilidad con la que cuenta esta escala es aceptable, con una puntuación de 0.82 en alfa de Cronbach (Cabañero et al., 2004). Esta versión consta de cinco ítems, con estructura similar a los anteriores instrumentos. Entre ellos por ejemplo, el ítem 3: <<Estoy satisfecho con mi vida >>.

La hoja de datos que recogía datos personales y sociodemográficos, incluyendo el sexo, edad, nivel de estudios, situación laboral, años en la organización, nacionalidad y organización a la que pertenecen los participantes, junto con los cuestionarios anteriores, conforma el cuaderno que se administró a los participantes.

Por último, se confeccionó también un consentimiento informado que informaba a los participantes de las condiciones de participación en la investigación, asegurando su seguridad y anonimato.

\section{Procedimiento}

La batería fue administrada tanto en formato físico, en las sedes de las organizaciones al principio de una reunión, como en formato digital, por correo electrónico. En este caso, el coordinador de la organización nos envió un correo con los resultados de sus voluntarios. A los participantes se les presentó el consentimiento informado antes de que cumplimentaran el cuestionario.

\section{Resultados}

Tras los análisis realizados, y como se muestra en la Tabla 1, los resultados han revelado que la edad correlaciona significativa y positivamente con desempeño cívico $(r=.52, p<.01)$ y con compromiso $(r=.30, p<.05)$. También se encontró que la edad correlaciona positivamente con los años en la organización $(r=.42 ; p<.01)$, es decir, las personas que empiezan el voluntariado a mayor edad, tienen más probabilidad de permanecer en la organización durante más tiempo. Además, los análisis correlacionales de las tres variables centrales han mostrado solamente una correlación positiva significativa entre desempeño cívico y compromiso organizacional $(r=.393$, $p<.01)$.

\begin{tabular}{|c|c|c|c|c|c|}
\hline \multicolumn{6}{|c|}{ Tabla 1. Correlaciones entre edad, AO, SV, CO, DC } \\
\hline & 1 & 2 & 3 & 4 & 5 \\
\hline 1. Edad & 1 & $0.42^{\star *}$ & 0.84 & $0.30^{*}$ & $0.52^{* \star}$ \\
\hline 2. AO & & 1 & -0.01 & 0.25 & 0.12 \\
\hline 3. SV & & & 1 & 0.22 & 0.15 \\
\hline 4. $\mathrm{CO}$ & & & & 1 & $0.39^{*}$ \\
\hline 5. DC & & & & & 1 \\
\hline
\end{tabular}


Por otra parte, tras la aplicación de una prueba $t$ de Student para encontrar posibles diferencias entre las variables, la división de la edad en dos rangos mostró efectivamente diferencias significativas $\left(t_{(47)}=-4.26, p<.01\right)$ en desempeño cívico, siendo el grupo de mayor edad (30 a 60 años) el que puntuaba más alto que los más jóvenes (18 a 29 años) $(M=4.57 ; 4.07)$. Sin embargo, no se han encontrado diferencias significativas entre hombres y mujeres en desempeño cívico $\left(t_{(47)}=-0\right.$ '893; N.S.), satisfacción vital $\left(t_{(47)}=-0\right.$ '6; N.S.) y compromiso organizacional $\left(t_{(47)}=-1\right.$ ' 41 ; N.S.).

Para comprobar si había diferencias significativas, se realizó una ANOVA sobre las variables desempeño cívico, compromiso organizacional y satisfacción vital, con el tipo de voluntariado como factor, pero los resultados no sugieren diferencias significativas en compromiso organizacional $\left(F_{(2,48)}=2\right.$ '92; N.S. $)$, desempeño cívico $\left(F_{(2,48)}=1\right.$ '46; N.S. $)$ o satisfacción vital $\left(F_{(2,48)}=0\right.$ '93; N.S.) entre los diferentes tipos de voluntariado contemplados en esta investigación. A modo exploratorio, cabe señalar que las medias en estas tres variables tienden a seguir una distribución de tal manera que el voluntariado en animales puntúa más alto en ambas tres, seguido de protección civil y teatro.

En esta misma línea, se llevó a cabo otra ANOVA con esas mismas tres variables, aunque con la experiencia como factor. La experiencia constó de tres niveles: voluntarios que contaban con de 1 a 3, de 4 a 7 , y de 8 a 22 años de experiencia en la organización. Se obtuvo una tendencia, aunque no significativa, donde la satisfacción vital desciende a medida que aumenta la experiencia $\left(F_{(2,48)}=0,27 ;\right.$ N.S. $)$, al contrario que el compromiso organizacional, que aumenta con la experiencia $\left(F_{(2,48)}=2\right.$ '67; N.S. $)$.

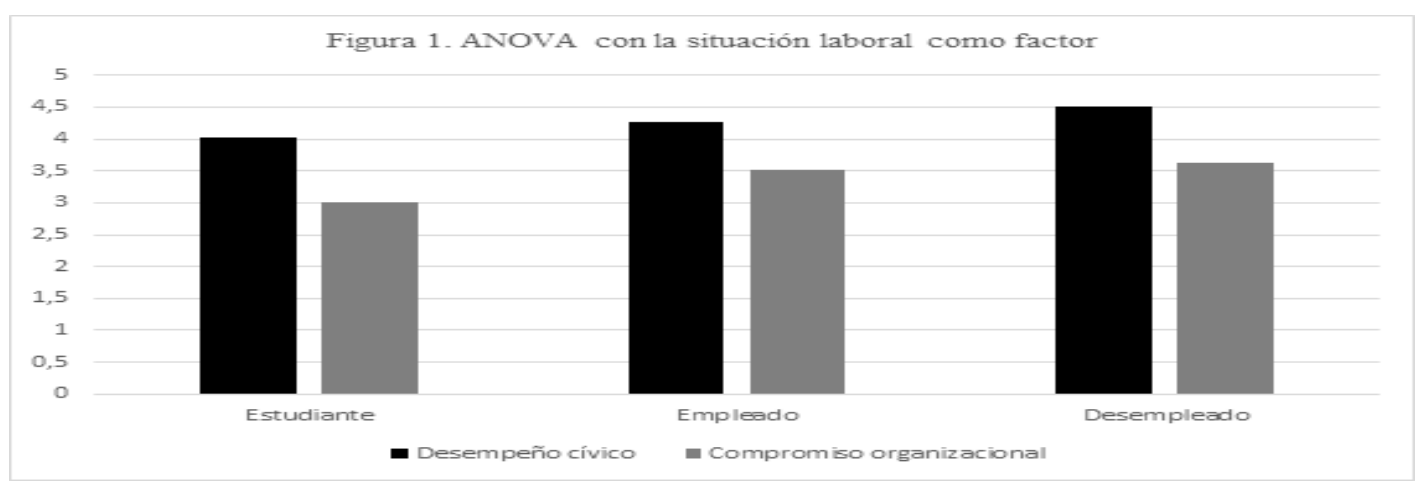

Por último, la realización de una ANOVA entre desempeño cívico y compromiso organizacional, con la situación laboral como factor, dio como resultado que los estudiantes puntúan más bajo que los desempleados en desempeño cívico $\left(F_{(2,48)}=5.59, p<.05\right)$. También se encontró que los estudiantes puntúan más bajo en compromiso organizacional que los empleados y los desempleados $\left(F_{(2,48)}=6.98\right.$; $p<.05)($ Ver Fig. 1).

\section{Discusión}

En relación a la hipótesis central de este estudio, tal y como se contempla en los resultados, no se encontraron diferencias significativas en las tres variables centrales con respecto al tipo de voluntariado, quedando así refutada dicha hipótesis. Estos datos contrastan con los de Dávila de León y Díaz Morales (2005), quienes encontraron diferencias en satisfacción vital entre sus dos tipos de voluntariado estudiados.

La correlación positiva que muestran las variables compromiso organizacional y desempeño cívico posiblemente guarde una relación con el hecho de que cuanto más 
comprometida e identificada se perciba una persona con su organización, será más probable que realice conductas ajenas a su rol que beneficien a la organización y a sus miembros. Para futuras investigaciones se sugiere estudiar con más detalle la relación entre estas dos variables y la conducta prosocial del voluntario.

Los resultados muestran que a medida que aumenta la edad aumenta también el desempeño cívico y el compromiso organizacional en los voluntarios. Es posible que esta relación esté modulada por el hecho de que más edad correlaciona con más años en la organización y con mayor compromiso, lo cual podría explicar también el aumento en desempeño cívico con la edad. Al igual que Dávila de León y Díaz Morales (2005), no se han encontrado diferencias significativas entre los grupos de edad en satisfacción vital.

Tal y como era de esperar, la falta de diferencias significativas entre hombres y mujeres en las variables centrales del estudio, concuerda con la literatura encontrada, aunque al no haber recabado una muestra homogénea en la distribución de sexo, los datos no son concluyentes.

De la misma manera y dado que la muestra está constituida únicamente por voluntarios, no podemos asegurar una correlación entre voluntariado y satisfacción vital tal y como Dávila de León y Díaz Morales (2005) y Grimm (2005) anteriormente encontraron en sus respectivos estudios.

A raíz de las tendencias señaladas en los resultados, y dado que las correlaciones y comparaciones en varios casos se encuentran próximas a la significación, se propone que para para futuras investigaciones se amplíe la muestra con intención de obtener unos resultados más fiables, y probablemente significativos.

Por último, se especificarán las limitaciones de este estudio que pueden haber influido en los resultados. En primer lugar, al estar la muestra formada únicamente por voluntarios, no se podrían establecer diferencias con población no voluntaria en ninguna de las variables. A este respecto, en futuras investigaciones debería incluirse un grupo control formado por población no voluntaria perteneciente a otro tipo de organizaciones, como empresas o partidos políticos.

En segundo lugar, dado el tamaño reducido de la muestra no sería recomendable inferir estos parámetros como poblacionales, careciendo este estudio por lo tanto de suficiente validez externa. En tercer lugar, ya que un gran número de voluntarios recibieron la petición de participar en la investigación vía correo electrónico, y sólo un número moderado de ellos cumplimentó los cuestionarios, es probable que este grupo esté sesgado ya que su mayor compromiso con la organización puede haber motivado su participación.

A pesar de las limitaciones anteriormente expuestas, los resultados indican que el desempeño cívico, la satisfacción vital y el compromiso organizacional no dependen únicamente del tipo de voluntariado en el que la persona se encuentre o de su sexo, sino que variables como la edad, la situación laboral o la puntuación de la persona en las tres variables anteriores están mediando el nivel de desempeño cívico, satisfacción vital y compromiso organizacional que muestra una persona en una organización de voluntariado. 


\section{Referencias}

Bateman, T.S., \& Organ, D.W. (1983). Job satisfaction and the good soldier: The relationship between affect and employee "citizenship". Academy of Management Journal, 26, 587-595.

Borman, W.C., \& Motowidlo, S.J. (1993). Expanding the criterion domain to include elements of contextual performance. En N. Schmitt, \& W. C. Borman (Eds.), Personnel selection in organizations (pp. 71-98). San Francisco, CA: Josey-Bass.

Cabañero Martínez, M.J. y Richart Martínez, M. y Cabrero García, J. y Orts Cortés, M.I y Reig Ferrer, A. y Tosal Herrero, B. (2004). Fiabilidad y validez de la escala de satisfacción con la vida de Diener en una muestra de mujeres embarazadas y puérperas. Psicothema, 16 (3), $448-455$.

Catano, V.M., Kelloway, E.K., \& Pond, M. (2001). Exploring commitment and leadership in volunteer organizations. Leadership \& Organization Development Journal, 22, 256-263.

Dávila de León, M.C. y Díaz Morales, J.F. (2005). Voluntariado y satisfacción vital. Intervención Psicosocial, 14, 81-94

Díaz Morales, J.F. y Sánchez-López, M.P. (2001). Relevancia de los estilos de personalidad y las metas personales en la predicción de la satisfacción vital. Anales de Psicología, 17, 151-158.

Díaz-Vilela, L., Díaz-Cabrera, D., Isla-Díaz, R., Hernández-Fernaud, E. y Rosales-Fernández, C. (2012). Adaptación al Español de la Escala de Desempeño Cívico de Coleman y Borman en el Análisis de la Estructura Empírica del Constructo. Revista de Psicología del Trabajo y de las Organizaciones, 28 (3), 135-149.

Diener, E. (1984). Subjective well-being. Psychological Bulletin, 95, 542-575.

Diener, E., Suh, E.M., Lucas, R.E., \& Smith, H.L. (1999). Subjective well-being: Three decades of progress. Psychological Bulletin, 125, $276-302$.

Diener, E., Emmons, R., Larsen, R., \& Griffin, S. (1985). The satisfaction with Life Scale. Journal of Personality Assessment, 49, 71-75.

Gaviria Stewart, E., Cuadrado Guirado, I. y López Sáez; M. (2009). Introducción a la psicología social. Madrid: Ed. Sanz y Torres / UNED

Grimm, N.J. (2005). Benevolence and well-being: the relationship of volunteering to life satisfaction. (Tesis doctoral en filosofia). The State University of New York, Buffalo.

Little, B. (1999). Personality and Motivation: Personal Action and the Conative Evolution. In Pervin, L.A., \& John, O. (Eds.), Handbook of Personality. Theory and Research (2nd ed) (pp. 501-524). New York: Guilford Press.

Meyer, J.P, \& Allen, N.J. (1997). Commitment in the workplace: Theory, research, and application. Thousand Oaks, CA: Sage Publications.

Mischel, W., \& Shoda, Y. (1995). A cognitive-affective system theory of personality: Reconceptualizing situations, dispositions, dynamics, and invariance in personality structure. Psychological Review, 102, 246-286.

Motowidlo, S.J. y Schmit, M.J. (1999). Performance assessment in unique jobs. In D.R. Ilgen, \& E.D. Pulakos (Eds.) The changing nature of performance: Implications for staffing, motivation, and development (pp. 56-87). San Francisco, CA: Jossey-Bass.

Nelson, M.C. (1997). Factors Related to Volunteer Commitment in Church-Related Services (Tesis de doctorado en trabajo social). The Catholic University of America, Washington, D.C.

Organ, D.W. (1988). Organizational citizenship behavior: The good soldier syndrome. Lexington, MA: Lexington Books.

Organ, D.W., \& Ryan, K. (1995). A meta-analytic review of attitudinal and dispositional predictors of organizational citizenship behavior. Personnel Psychology, 48, 775-802.

Penner, L. (2002). Dispositional and Organizational Influences on Sustained Volunteerism: An Interactionist Perspective. Journal of Social Issues, $58,447-460$.

Porter, L.W., Steers, R.M., Mowday, R.T., \& Boulian, P.V. (1974). Organizational commitment, job satisfaction, and turnover among psychiatric technicians. Journal of Applied Psychology, 59, 603-609.

Ployhart, R.E., Schneider, B., \& Schmitt, N. (2006). Staffing organizations. Contemporary practice and theory (3ªed.). Mahwah, NJ: Lawrence Erlbaum Associates. 
Salmela-Aro, K., \& Nurmi, J-E. (1997). Goal contents, well-being and life context during transitions to university: a longitudinal study. International Journal of behavioral development, 20, 471-491.

Strack, F., Argyle, M., \& Schwart, N., (1991). Subjective well-being: An interdisciplinary perspective. International Series in Experimental Social Psychology, n 21, Pergamon Press.

Veenhoven, R. (1991). Questions on happiness. Pergamon press.

Washko, M.M. (2006). Volunteering \& Older Adult: An Exploration of Behavior Determinants and their Effects on Volunteer Commitment. Doctoral Thesis of the University of Massachusetts, Boston.

Witman, D.S., Van Rooy, D.L., \& Viswesvaran, C. (2010). Satisfaction, citizenship behaviors, and performance in work units: A meta-analysis of collective construct relations. Personnel Psychology, 63, 41-81. 